\title{
Pain management barriers in critical care units: A qualitative study
}

\author{
Abdul- Monim Batiha \\ Faculty of Nursing, Philadelphia University, Jordan \\ E-mail: abatiha@philadelphia.edu.jo
}

\begin{abstract}
Background: Uncontrolled pain in intensive care units triggers physical and emotional stress responses, inhibits healing, increases the risk of other complications, and increases the length of ICU stay.

Aim: To explore pain management barriers as identified by Jordanian critical care units.

Method: This study employed qualitative content analysis with 37 participant nurses from Jordanian critical care units. Data were obtained through semi-structured serial interviews. Purpose nonprobability sampling was used for the initial interviews.

Results: Several themes emerged to describe barriers to managing pain identified by the critical care nurses. These were grouped into three main themes: (1) Barriers related to patients with subgroups such as patient did not want to bother nurses, patients' difficulty with completing pain scales, patients' reluctance to take pain medications because of side-effects, patients reporting their pain to the doctor, but not to the nurse, and fatalistic beliefs. (2) Barriers related to nurses that included patient sedation, frequent complaints from patients, inconsistent practices around administering if necessary medications, time limitations, limited communication, the fear of side effects of pain drugs, physicians' lack of trust in the nursing assessment of pain in critically ill patients, inadequate staff knowledge of pain management, and fear of causing delirium or confusion. and (3) Barriers related to hospital policies includes: policies and rules of hospital, lack proper pain assessment tool, nursing shortages, powerlessness, interruptions of activities relating to pain, lack of psychosocial support services, lack of alternatives non-pharmacologic therapy, and lack of pain management drugs.

Conclusion: The results identify potential pain management barriers which can be considered when developing and disseminating policies and procedures in managing the pain in Jordanian critical care settings.
\end{abstract}

Keywords: barriers, critical care units, Jordan, nursing, pain management, Qualitative study.

\section{Introduction}

Uncontrolled pain in intensive care units triggers physical and emotional stress responses, inhibits healing, increases the risk of other complications, and increases the length of ICU stay (Morton et al. 2005). Pain is the greatest concern of patients in intensive care units (ICUs). Most critically ill patients experience moderate to severe pain (Halm et al. 1993, Fralic 1999, Batiha et al 2013). The critically ill patient is particularly susceptible to the negative effects of untreated pain. Discomfort related to invasive tubes, diagnostic tests, as well as post-operative pain is common for the critical care patient. Pain for these patients can cause increased sympathetic nervous activity, ventilator dyssynchrony, and cardiovascular events (Wong et al. 2004).

The intensity of procedural pain experienced may vary between individuals. It is influenced by a number of factors. These include the patient's previous experience and existing of pain, procedure to be performed and its duration, patient's possible fear, anxiety and expectations of pain associated with the procedure (Ashley \& Given 2008).

Pain and its management have been a fairly low priority for receiving time and attention from healthcare professionals, and nurses seemed to place a lower priority on activities that directly impacted patient comfort, such as administering analgesics in an appropriate time frame (Manias 2003, Richards \& Hubbert 2007). Efforts to provide pain relief and comfort measures are complicat- ed by the fact that critical care nurses must continuously perform procedures or treatments that cause pain to the patient. Procedures like chest tube insertion and removal, endotracheal suctioning, and wound debridement are obviously painful. Recent research reveals that simple and routine procedures, such as turning the patient, can also cause considerable pain (Morton et al. 2005, Puntillo 2001, Thompson 2001). Nurses play a crucial role in pain assessment and management. They often act as mediators between the doctor and the patient, and serve as the main observer of pain and discomfort in the latter. Therefore, it is very important to determine the barriers perceived by nurses to the assessment and management of pain.

Despite the fact that nurses are the front-line caregivers in the inpatient settings, there is no qualitative research on barriers regarding pain management in Jordanian critical care units, so this research addresses this research gap.

\section{Methods}

\subsection{Method and participants}

This study employed qualitative content analysis with 37 participant nurses from Jordanian critical care units. Data were obtained through semi-structured serial interviews. Purpose sampling was used for the initial interviews, a nonprobability sampling method in which participants were selected based on the researchers' per- 
sonal judgment about which nurses would be most representative of critical care nurses in Jordan. According to the emerging codes and categories data were collected by means of theoretical sampling. Sampling was targeted based on a set of predetermined criteria. The researchers made preliminary sampling decisions to select staff with a minimum of two years' nursing experience working in critical care units in two teaching hospitals in Jordan. It was considered that the participants would have sufficient work experience to enable them to analyse pain management barriers in critical care units. The sampling was based on the maximum variant approach. Sampling started with a nurse of 27 years' experience presented by the ICU head nurses and then with selection by snowballing technique, whereby participants were asked to suggest the names of other participants. Sampling thus extended to other nurses. Data collection and analysis proceeded concurrently with the development of themes related to the reality of the nurses' perceptions of barriers regarding pain management in critical care units. Sampling continued until saturation was reached; this was when no new categories or subcategories emerged.

\subsection{Data collection}

Upon agreeing to participate in the research, and after signing the informed consent sheet, nurses were given an appointment for the interview. Individual semi-structured interviews were conducted in a private room at the assigned hospital. Permission for taperecording the interviews was obtained from each participant. The interviews lasted from 15 to 30 minutes. The interview guide consisted of core open-ended questions to allow the respondents to explain their own viewpoints and experiences as comprehensively as possible. In congruence with the qualitative methodology, the opening question for each interview was "talk about anything you considered important regarding pain management barriers in Jordanian critical care units".

\subsection{Data analysis process}

Data analysis started concurrently with data collection and each interview was transcribed exactly and analyzed before the next interview took place. The process of interviewing was stopped when data saturation occurred. The author transcribed the taperecorded interviews and the data were analyzed using the method of content analysis (Sandelowski 2000).

The analysis started by identifying the units of meanings that could be extracted from the statements, which were essential for participants' experiences. It proceeded using line-by-line coding; codes were freely generated during repeated discussions between the researchers. Statements that were unrelated to the study were excluded. Codes with similar meanings were grouped into categories. The transcripts were reviewed in order to validate the codes and categories. Regarding trustworthiness, credibility was established through peer check.

The participants were contacted after the analysis and were given a full transcript of their respective coded interviews with a summary of the emergent themes to determine whether the codes and themes matched their experiences. Then the researcher conducted the peer checking. Prolonged engagement with the participants within the research field helped the main researcher to gain the participants' trust and a better understanding of the research fields Maximum variation of sampling (in terms of the type of ward, years of working experience and place of duty) also enhanced the conformability and credibility of data. This sampling strategy enabled the researcher to capture a vast range of views and experiences (Streubert \& Carpenter 2003). The analysis was finalized by identifying several themes that emerged to describe barriers regarding pain management in Jordanian critical care units.

\subsection{Ethical issues}

The ethical committees at the participating hospitals in this study approved the research proposal. Ethical issues were concerned with the participant's autonomy, confidentiality and anonymity during the study period. All participants were informed of the purpose and design of the study and also the voluntary nature of their participation. Informed consent was obtained from the participants in writing and signed by them for all stages of the study.

\section{Results}

\subsection{General characteristics of the participants}

The participants' ages ranged from 24 to 55, with an average of 34.1 years. Nursing practice experiences ranged from 2 to 27 years, with a mean of 11.2. Thirty-two of participants had graduate degree in nursing, and five participants had postgraduate qualifications. Nineteen participants were female and eighteen were male.

\subsection{Pain management barriers}

The chief barriers identified by the nurse participants in this study were those related to patients, to nurses and to hospital policies. The salient points are listed below, along with relevant data (i.e. quotations of participants). Statements are from either ICU ward nurses or from ICU head nurses (where indicated).

\subsubsection{Barriers related to patients}

1) Patients did not want to bother nurses:

"Many patients are reluctant to report pain because they did not want to disturb me".

2) Patients' difficulty with completing pain scales (e.g. 0-10):

"Although it is rare to use pain scale, when I use it the patients have difficulty in using it".

3) Patients' reluctance to take pain medications because of sideeffects:

"Many patients refuse to take pain medications because they are afraid of its side-effects".

4) Patients reporting their pain to the doctor, but not to the nurse:

"Our patients trust doctors more than nurses; they tell them their complaints more than us".

5) Fatalistic beliefs, which induce lack of self-motivation to comply with prescribed medical treatment:

"Some patients told me that health and illness are from God, nobody can prevent it, and we are not capable of influencing the disease and pain in any way".

"This pain is test from God; I will bear it to win credit from my God".

\subsubsection{Barriers related to nurses}

1) Patient sedation acts as a pain management barrier.

"My patient is sedated; thus, I cannot assess his pain".

2) Frequent complaints from patients are another barrier to pain management:

"Some patients complain too frequently so I can't judge if pain is real".

3) Inconsistent practices around administering if necessary (prn) medications:

"The decision to administer [prn] medication for pain medication is up to the assigned nurse, and varies from one to another".

4) Time limitations:

"We cannot spare time for the patients even if we want to, because we have a lot of working in a limited time".

5) Limited communication was also viewed as an important barrier for nurses' role as comforters:

"I complain of low communications level between me and the patient".

"There is low level of communication between me and doctors, which leads to negative management of pain for our patients".

6) The fear of side effects of pain drugs: 
"When doctors order morphine injection [prn], I will hesitate to give it because I fear its side-effects like addiction, respiratory depression, or death".

7) Physicians' lack of trust in the nursing assessment of pain in critically ill patients:

"There is no trust from physician to my pain assessment, documentation, and report".

8) Other barriers due to lack of pain management knowledge about pain management in infants, children and elderly:

"From my observation, my staff thinks that infants don't feel pain, and cognitive impairment blunts the perception of pain in the elderly". (Head nurse)

9) Inadequate staff knowledge of pain management:

"Although pain management is very important, unfortunately many staff did not have enough knowledge about it". (Head nurse)

10) Fear of causing delirium or confusion is a barrier to pain management for the elderly:

"If I give pain management to elderly patients they will suffer from delirium or confusion".

\subsubsection{Barriers related to hospital policies}

1) Several nurses noted that "policies and rules of hospital" act as barriers to pain management. Comments that reflected this include:

"My hospital policies and rules acts as barriers to pain management of critically ill patients. There are no obvious policies, rules and guidelines to deal with patients 'pain".

2) Lack proper pain assessment tool:

"My hospital lacks pain assessment tool to be used in our clinical practice".

3) Nursing shortages (patient-to-nurse ratio) is another barrier to pain management:

"Our critical care unit are not following the standard of universal patient to-nurse ratio so this lead to time constrain, decrease quality of care".

4) Participants cited "powerlessness" as administering proper pain prescription requires doctors' orders. This was considered as a key barrier to pain management:

"Now, it is expected that we [nurses] only following doctor orders for everything concerning pain management".

5) Another theme that emerged from the data collected was "interruptions of activities relating to pain".

"Frequently, many activities interrupted me when carrying out activities relating to pain, like doctors' and family members' questions, alarms, telephone... etc."

6) Lack of psychosocial support services:

"There are no psychosocial support services in our hospital; it's limited to some cancer patients in oncology units".

7) Lack of alternatives non-pharmacologic therapy for pain management (e.g. cold and hot compresses acupuncture):

"Although I heard about non-pharmacologic therapy for pain management, actually I always use drugs like morphine or pethidine for pain management".

8) Lack of pain management drugs:

"Sometimes there is a shortage of drug supply for pain management in my unit".

\section{Discussions}

This study is the first to understand barriers regarding pain management in Jordanian critical care units. The factors that were identified as pain management barriers and their mean item ratings indicated that major barriers originated from three sources: patients, nurses and hospital policies. Among the patient-related barriers, some patients did not want to bother nurses and are reluctant to report pain; others have no adherence to treatment regimens, fear of dependency, anxiety over being a "bad patient", concerns about disturbing health care providers, and fear of sideeffects. These findings are in-line with previous studies (Halm et al. 1993, Sandelowski 2000, Streubert \& Carpenter 2003, Erdek \& Pro-novost 2004).

Giving a proper pain prescription needs a doctor's approval, according to hospital policy. Nurses cannot depend upon their own pain assessment to prescribe pain medicine. These barriers for Jordanian nurses demonstrate that nurses' pain management practice can be influenced by national policies and culture. In addition, current pain assessment forms in Jordanian ICUs focus on cancer pain management (Batiha 2012). There are no suitable painassessment forms for ICU patients who cannot report pain. This barrier is similar to that reported for ICU nurses in the United States (Erdek \& Pronovost 2004).

Pain relief is often relegated to a low priority owing to the lifethreatening nature of the patient's illness and the other lifesaving interventions that are required in ICUs. Critical care nurses are often concerned that analgesic administration may create problems, such as hemodynamic and respiratory compromise, over sedation, or drug addiction. With the regulation and controlled dispensing of opioids, fear of opioids should not be as common as it evidently is in Jordanian health care settings (Tawalbeh et al 2013), among both patients and health care providers. Unfortunately, some over-cautious and misinformed health care professionals still suffer from opiophobia, which results in inadequate pain management. Professionals' discomfort in administering opioids is usually related to concerns of causing addiction, overdose, respiratory depression, or death. However, the incidence of addiction after using opioids for pain management is less than $1 \%$ (Joranson et al. 2000). A recent study showed that despite the increased medical use of strong opioids such as morphine and hydromorphone, abuse levels have not exceeded 1\% (Joranson et al. 2000).

A number of practitioners have concerns regarding opioids being diverted for recreational use. In fact, the data suggest that opioid analgesia is a relatively small part of drug abuse. Of drugs used for illicit purposes, opioids are used in only $3.8 \%$ of cases, and the drugs most frequently abused recreationally are alcohol, amphetamines and sedatives (Joranson et al. 2000).

Other barriers due to lack of knowledge affect pain management in infants and children. There is a misconception among some health care professionals that infants do not feel pain. In fact, the nervous system in children and infants can be more sensitive than in adults (Howard 2003, Heather 2005, Batiha \& Bashtawy 2013). This is clinically significant, as lower thresholds of mechanical and thermal stimuli could result in a greater perception of painful stimulus in children than would occur in adults (Howard 2003). There remains a lack of confidence in prescribing and administering pain medication to pediatric patients (Norton et al. 2002). The elderly are also a group that often receives inadequate pain management.

There is a misconception among some practitioners that cognitive impairment blunts the perception of pain. Although the elderly may be more sensitive to the effects of opioids, there is no reason to withhold analgesia or to under-treat pain. Instead, one should assess the individual, and start with low doses of analgesia.

Fear of causing delirium or confusion is a barrier to pain management for the elderly. This result concurs with the findings of Allen et al. (2003), that fewer than $25 \%$ of elderly post-operative patients received their mean level of prescribed opioid analgesia.

A poor pain assessment is another barrier to pain management. Assessments may not be thorough or may be performed less frequently than necessary. There may be a lack of validity given to the patient's self-reporting, which may be tied to inadequate knowledge on pain and pain management (Ekman \& Koman 2004, Hsiang-Ling \& Yun-Fang 2010) Use of a standard pain rating scale facilitates consistency in pain assessments.

The results of this study indicate that nurses perceive a variety of barriers when attempting to provide optimal pain assessment and management in ICU. Lack of psychosocial support services was the most commonly perceived barrier related to hospital policies (Tawalbeh et al 2013). This finding is similar to the results of study by Furstenberg et al. (1998) which defined the lack of sup- 
port systems as being a barrier interfering with optimal pain management. In assigned hospitals, psychosocial services are not part of routine patient care. Although psychological support can be provided to a very limited number of patients with cancer upon the request of the attending physician, there is no social work for patients. Institutional and governmental attempts are needed to establish psychosocial support services for all patients. Another commonly expressed hospital policies barrier was the patient-tonurse ratio. This result was also consistent with some earlier studies (Anthony 1999, Ekman \& Koman 2004).

When the patient-to-nurse ratio is high, nurses experience time constraints which interfere with quality of care. Research from developed countries differs from this finding; Johnson et al. (2000) reported that only $13 \%$ of nurse's identified time as a barrier to pain management. However, understaffing remains a barrier to optimal patient care in Jordan. Each nurse has to take care of 8 to 10 patients in the hospital where this study was conducted.

The appropriate assessment and treatment of pain is highly dependent upon communication between physicians and nurses. Lack of adequate and accurate communication between nurses and physicians was reported as an important barrier to optimal management of pain. Van Niekerk and Martin (2003) showed that nurses who did not feel adequately consulted by physicians were significantly more likely to encounter barriers such as insufficient cooperation from patients' physicians and inadequate prescription of analgesic medications.

Physicians' lack of trust in the nursing assessment of pain in critically ill patients was another perceived barrier. This shows the negative impact of lack of communication between the nurses and doctors. The nurses indicated that they had difficulty contacting or communicating with physicians to discuss treatment of pain. It has been reported that the barriers to effective pain management encountered by nurses were affected by their relationship with physicians (Van Niekerk \& Martin 2003, Van Niekerk \& Martin 2002). Teamwork is essential for providing good-quality pain management in hospitals. It is important to establish a supportive team spirit between doctors and nurses. For this, meetings should be held between these two groups of health professionals to facilitate the discussion of pain management problems and to review recommendations for solutions. There are studies showing that education is effective in eliminating the barriers standing in the way of the evaluation and management of pain. For this reason, there should be regular and continuous education programs for all health professionals who are involved in the pain management We need to define the knowledge and attitude of nurses and doctors in our hospital with an aim to set up a continuous education program on pain management. In addition, evidence-based pain management guidelines should be drawn up for the clinical use of doctors and nurses.

\section{Limitations}

Although the data provided a rich description of barriers regarding pain management in Jordanian critical care units from the nurse participants' viewpoint, generalization of research findings to the larger population of nurses is limited.

Results were, however, checked with several expert nurses who did not participate in the research and they confirmed the fitness of the results. Comments provided by these nurses also support the transferability of the findings, at least within the context of the Arab/Middle-Eastern healthcare context. As in all self-reported research, the findings now need to be supported by detailed observational studies.

\section{Conclusions and recommendations}

The findings of this study will be helpful for clinicians to better develop policies and procedures using clinical practice guidelines. The results identify potential barriers which can be considered when developing and disseminating policies and procedures in managing the pain in Jordanian critical care settings. Appropriate strategies to minimize potential barriers can be developed to better enable nurses to implement practice guidelines to generate effective pain management in critically ill patients.

There is a pressing need to update the present curriculum of nursing and medical programs to include updated strategies for pain relief in critically ill patients. Drawing from the study results, the researcher recommends that ICU nurses should be provided with ongoing pain management courses. This education should include topics such as pain symptoms, pain assessment, mechanisms of analgesia, evaluation of analgesia, effects and side-effects of pain medications and nonpharmacological pain management. At the same time, nurses' knowledge and pain management ability should be enhanced by using case studies and related courses should be regularly updated.

\section{Acknowledgement}

We wish to thank all the nurses who participated in this study and the patients who made these experiences possible, as well as the other hospital staff who facilitated this research.

\section{References}

[1] Allen R., Thorn B., Fisher S., et al. (2003), Prescription and dosage of analgesic mediation in relation to resident behaviors in the nursing home. Journal of the American Geriatrics Society 51 534-538.

[2] Anthony MK (1999), the relationship of authority to decision-making behavior: implications for redesign. Res Nurs Health 22 388-98.

[3] Ashley E, Given J (2008), Pain management in the critically ill. Journal of Perioperative Practice 1811 504-509.

[4] Aslan FE, Badir A \& Selimen D (2003), how do intensive care nurses assess patients' pain? Nursing in Critical Care 8 62-67.

[5] Batiha A-M \& AL Bashtawy M (2013), Knowledge of Philadelphia University students regarding blood Donation. Transfusion Medicine 23, 195-198.

[6] Batiha A-M (2012), Pain Management Barriers in Jordanian Critical Care Units. International Journal of Advanced Nursing Studies 1(2), 73-83.

[7] Batiha A-M, Bashaireh I, Albashtawy M \& Shennaq S (2013), explores the competency of the Jordanian intensive care nurses towards endotracheal tube and oral care practices for mechanically ventilated patients: an observational study. Global Journal of Health Science 5, 203-213.

[8] Ekman E, Koman A (2004), acute pain following musculoskeletal injuries and orthopaedic surgery. The Journal of Bone and Joint Surgery 86-A 1316-1324.

[9] Erdek MA \& Pronovost PJ (2004), Improving assessment and treatment of pain in the critically ill. International Journal for Quality in Health Care 16 59-64.

[10]Fralic MF (1999), Nursing leadership for the new millennium: Essential knowledge and skills. Nurs Health Care Perspect 20(5) 260-265

[11]Furstenberg CT, Ahles TA, Whedon MB, et al. (1998), Knowledge and attitudes of healthcare providers toward cancer pain management: A comparison of physicians, nurses, and pharmacists in the state of New Hampshire. Journal of Pain and Symptom Management 15 335349.

[12] Halm MA, Titler MG, Kleiber C, et al. (1993), Behavioral responses of family members during critical illness. Clin Nurs Res 2 414-437.

[13]Heather E (2005), Improving pain management for critically ill and injured patients. Canadian association of critical care nurses. 16 (3), 26-31.

[14]Howard R (2003), Current states of pain management in children. The Journal of the American Medical Association 290 2464-2469.

[15]Hsiang-Ling W, Yun-Fang T (2010), Nurses' knowledge and barriers regarding pain management in intensive care units. Journal of Clinical Nursing $193188-3196$.

[16]Joranson D.E., Ryan, K.M., Gilson, et al. (2000), Trends in medical use and abuse of opioid analgesics. The Journal of the American Medical Association 290 1710-1714

[17]Krairiksh M, Anthony MK (2001), Benefits and outcomes of staff nurses' participation in decision-making. J Nurs Adm 31 16-23.

[18]Manias E (2003), Pain and anxiety management in the postoperative gastro-surgical setting. J Adv Nurs 41 585-94. 
[19]Morton P, Fontaine D, Hudak C, et al. (2005), Relieving pain and providing comfort, Critical care nursing: a holistic approach. USA: Lippincott Williams \& Wilkins, 8th edition, 46-61.

[20]Norton L, Gundersen B, Pitcher K. (2002) Children and pain medications: The pharmacist's view. The Pain Practitioner 12(4) 4-8.

[21]Puntillo KA, White C, Morris A, et al. (2001), Patients' perceptions and responses to procedural pain: Results from Thunder II Project. Am $J$ Crit Care 10(4) 238-251.

[22]Richards J, Hubbert AO (2007), Experiences of expert nurses in caring for patients with postoperative pain. Pain Manag Nurs 8 17-24.

[23] Sandelowski M (2000), whatever happened to qualitative description? Research in Nursing and Health 19 114-123.

[24] Streubert HJ, Carpenter DR (2003), Qualitative Research in Nursing Advancing the Humanistic Imperative. 3rd edn. Lippincott, Philadelphia, PA.

[25] Tawalbeh L, Ahmad Tubaishat A, Batiha A-M, Manar Al-Azzam M \& AlBashtawy M (2013), The Relationship Between Social Support and Adherence to Healthy Lifestyle Among Patients With Coronary Artery Disease in the North of Jordan. Clinical Nursing Research. DOI: $10.1177 / 1054773813501194$.

[26] Thompson CL, White C, Wild L, et al. (2001), Translating research into practice: Implications of Thunder Project II. Crit Care Nurs Clin North Am 13(4) 541-546.

[27]Van Niekerk LM, Martin F (2002), the Impact of the Nurse-Physician Professional Relationship on Nurses' Experience of Ethical Dilemmas in Effective Pain Management. Journal of Professional Nursing 18 (5) 276-288.

[28] Van Niekerk LM, Martin F (2003), the Impact of the Nurse-Physician Relationship on Barriers Encountered by Nurses during Pain Management. Pain Management Nursing 4 (1) 3-10.

[29]Wong C, Burry L, Molino-Carmuna S, et al. (2004), Analgesic and sedative pharmacology in the intensive care unit. Dynamics 15(1) 23 - 